\title{
Wharton's Jelly Stem Cells as Agents for Cancer Therapy
}

\author{
Masaaki Tamura ${ }^{*}$, , Atsushi Kawabata ${ }^{1}$, Naomi Ohta ${ }^{1}$, Lakshmi Uppalapati ${ }^{1}$, Kevin G. Becker $^{2}$ and \\ Deryl Troyer ${ }^{1}$
}

\author{
${ }^{I}$ Departments of Anatomy and Physiology, Kansas State University, Manhattan, KS 66506, USA \\ ${ }^{2}$ Gene Expression and Genomics Unit, NIH Biomedical Research Center, National Institute on Aging, NIH, Baltimore, \\ MD 21224, USA
}

\begin{abstract}
Stem cell based therapy has significant potential to treat various diseases including primary and metastatic cancers. The umbilical cord matrix stem cells (UCMSC) derived from human umbilical cord Wharton's jelly (also termed WJMSC) have been shown to exhibit low immunogenicity, which potentially negates immune consequences after cytotherapy. The homing ability of human and rat UCMSC to inflammatory tissues, including cancer tissues, further confers upon these cells the potential for targeted cancer therapy. Our previous studies demonstrated that un-engineered human and rat UCMSC significantly attenuated the growth of multiple cancer cell lines in vivo and in vitro through multiple mechanisms. We have also demonstrated that these cells can be engineered to express cytotoxic cytokines before being delivered to the tumor and can be preloaded with nanoparticle payloads and attenuate tumors after homing to them. In this review, intrinsic stem cell-dependent regulation of cancer growth, potential mechanisms involved in this unique biological function, delivery of exogenous anti-cancer agents, and the potential for clinical applications will be discussed.
\end{abstract}

Keywords: Cytotherapy, Immunogenicity, Tumor homing, Tumor tissue tropism, Umbilical cord matrix stem cell, Wharton`s jelly.

\section{INTRODUCTION}

Wharton's jelly is a gelatinous tissue within the umbilical cord that contains myofibroblast-like stromal cells. These stromal cells originate from extra-embryonic mesoderm which is derived from the epiblast [1-3]. Wharton's jelly is largely made up of mucopolysaccharides (hyaluronic acid and chondroitin sulfate). First named by Thomas Wharton in 1656, this gelatinous substance's function is to provide support and cushioning for the vein and arteries of the umbilical cord. The primitive mesenchymal-like cells of Wharton's jelly are trapped at a very early embryological age and retain the properties of primitive stem cells. Stem cells are suggested to possess the ability to self renew throughout life to maintain the stem cell population and the capacity to differentiate to become specialized progeny cells. Cells in Wharton's jelly express several stem cell characteristic genes, and have a high telomerase activity $[4,5]$. They possess stem cell properties and can be more easily obtained and processed than embryonic and mesenchymal stem cells (MSC) such as bone marrow-derived stem cells (BMMSC) $[6,7]$. Although research shows the potential of umbilical cord matrix stem cells (UCMSC) for therapy in nerve injuries, diabetes mellitus, and several organ type cancers, the impact of naive UCMSC on tumorigenesis and tumor growth has not yet been established.

Regarding the impact of MSC from sources other than umbilical cords on tumorigenesis, several studies have shown that MSC from other sources promote tumor growth

*Address correspondence to this author at the Departments of Anatomy and Physiology, Kansas State University, Manhattan, KS 66506, USA; Tel: 785532-4824; Fax: 785-532-4557; E-mail: mtamura@vet.ksu.edu and metastasis by multiple mechanisms [8-10]. Furthermore, it has been revealed that BMMSC induce many pro-tumorigenic effects, such as host immunosuppression [11], tumor vascular formation [12], and reinforcement of the epithelialmesenchymal transition [13]. In allogeneic transplantation models, MSC-dependent immune suppression plays a critical role; thus, tumors are frequently formed, but only in the presence of embryonic, BMMSC [14]. On the other hand, a few studies report that MSC from sources other than umbilical cords can suppress tumor growth. For example, Khakoo et al., reported that BMMSC could exert anti-tumor effects on Kaposi's sarcoma, but only if there was cell-cell contact [15]. In this review, the intrinsic tumoricidal ability of naïve UCMSC, potential mechanisms involved in this unique biological function, delivery of exogenous anticancer agents, and the potential for clinical applications will be discussed.

\section{POTENTIAL APPLICATION OF UCMSC FOR VARIOUS DISEASES}

Among the features of UCMSC, they have high proliferative potential and differentiation capacity into cells of multiple mesenchymal lineages. For example, Yang et al. have shown that transplantation of human UCMSC into the lesion site of a spinal cord injury is beneficial to wound healing in rats [16]. Parkinson's disease is a potential target for the UCMSC therapy since transplanted UCMSC can be differentiated into dopaminergic neuron-like cells [17]. In addition, Chao et al., have reported that human UCMSC can be differentiated into mature islet-like cell clusters, and they possess an insulin-producing ability and significantly alleviated diabetic conditions in rats [18]. Taken together, 
these results indicate that UCMSC could be used for multiple targeted therapies including cancer therapy.

\section{UCMSC POSSESS LOW IMMUNOGENICITY}

Although UCMSC are relatively easy to prepare in large quantities and can be banked until use, their practical application would be in allogeneic transplantation. One important requirement for allogeneic cell transplantation therapy is low immunogenicity. UCMSC apparently escape the host immune responses by multiple mechanisms. They express MHC class I (HLA-ABC) but not class II (HLA-DR) antigens. They are negative for other co-stimulatory antigens such as CD80 and CD86 [5, 19, 20]. This characteristic is similar to other MSC, since MSC are generally MHC class II antigen negative [21]. UCMSC produce large amounts of tolerogenic IL-10 and TGF- $\beta$ and express HLA-G at higher levels than BMMSC $[5,20,22]$. HLA-G induces the expansion of regulatory $\mathrm{T}$ cells (Treg), which would contribute to the suppression of effector responses to alloantigens [23, 24]. It has also been shown that UCMSC suppress $T$ cells and NK cells by stimulating Treg activation [22] and expressing Nanog and Oct3/4A [5]. UCMSC showed very weak $\mathrm{T}$ cell activation with non-significant increases in IFNgamma and IL-2 [22]. Furthermore, UCMSC derived prostaglandin E2 (PGE2) modulates monocytes to suppress $T$ cells $[25,26]$. Adhesion molecules, such as inflammatory cytokine-induced intercellular adhesion molecule-1 (ICAM1) [20] and vascular cell adhesion molecule-1 (VCAM-1), play critically important roles for the MSC-dependent immunosuppression of $\mathrm{T}$ cells. These molecules are inducible by the parallel presence of IFN- $\gamma$ and inflammatory cytokines such as IL-1 and TNF- $\alpha$ [5, 20]. Furthermore, UCMSC express IL-6 and vascular endothelial cell growth factor (VEGF), molecules which are thought to suppress the immune system [21]. The immunosuppressive properties of UCMSC are significantly stronger than those of BMMSC. In fact, un-stimulated UCMSC suppress mitogen-induced lymphocyte-proliferation responses to a greater extent than BMMSC. In a co-culture of 1:100 UCMSC:PBMC, suppression of lymphocyte proliferation responses was observed [20]. Additionally, priming with inflammatory stimuli can enhance only BMMSC, not UCMSC, to stimulate mitogeninduced lymphocyte proliferation [20]. These UCMSC characteristics are very important and convenient properties as these cells are applied to cytotherapeutic treatment of various diseases including cancer.

Despite the immunosuppressive properties of UCMSC, repeated injections of UCMSC could induce MHC class II
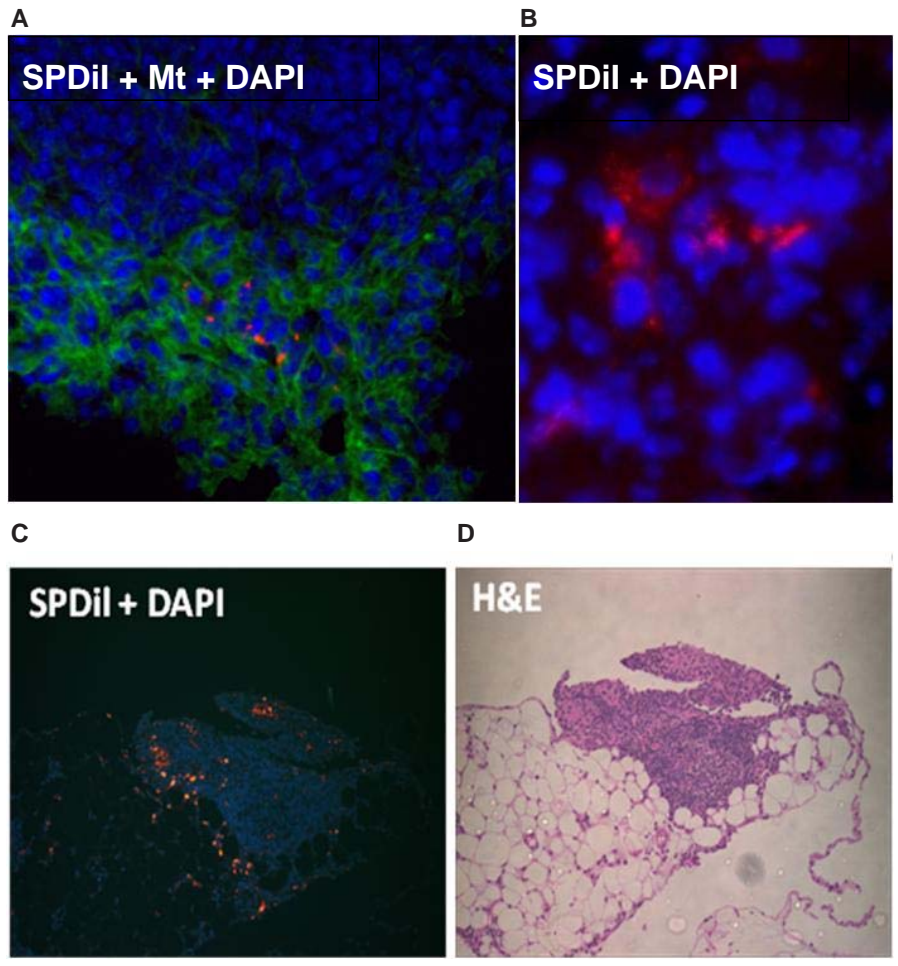

Fig. (1). Transplanted human or rat UCMSC exhibit tumor tropism. Panels A and B, MDA-MB 231 lung metastatic breast carcinomabearing SCID mice were treated systemically via intravenous (IV) injection with human UCMSC labeled with SpDiI red fluorescent dye (5 x $10^{5}$ cells) [32]. One week after the last treatment, lungs or pancreatic tumors were dissected and subjected to immunohistochemical analysis. Sections were counterstained with Hoechst 33342 nuclear stain (DAPI, blue). Panel A, MDA-MB 231 cells in the lung were identified by anti-human mitochondrial antibodies conjugated with FITC (Mt, green) to pinpoint the location of SpDiI labeled human UCMSC within the tumor. Panel B, Morphology of SP-DiI-labeled cells in the lung section indicated that the SP-DiI-labeled cells appear to be UCMSC. Panels $\mathrm{C}$ and D, PAN02 murine pancreatic carcinoma sections from peritoneal tumors of mice treated via intraperitoneal injection with rat UCMSC labeled with SP-DiI $\left(5 \times 10^{5}\right.$ cells) [33]. Sections were visualized with the fluorescent microscope or in bright field after H\&E staining. These figures clearly indicate that systemically or intraperitoneally administered human or rat UCMSC selectively engrafted within the tumors or in close proximity to the tumors. Scale bar $=100 \mu \mathrm{m}$. Figure is modified from the original figures, Rachakatla et al., Cancer Gene Ther, 14: 828-835 2007, and Ayuzawa et al., Cancer Lett, 280: 31-37, 2009. 
antigen expression [21]. It is also shown that $48 \mathrm{~h}$ exposure to IFN- $\gamma$ induces MHC class II antigen and increases MHC class I antigen expression in vitro [21]. These results imply that repeated injection or prolonged exposure to IFN- $\gamma$ may increase the immunogenicity of UCMSC. In addition, differentiation of UCMSC may decrease their immunosuppressive properties [21]. In BMMSC, chondrogenic differentiation has also been suggested to increase immunogenicity [27], and the same could hold true for UCMSC. Although the relationship between differentiation and immunogenicity of MSC is still unsettled, these reports suggest that the low immunogenicity of UCMSC, which is an important characteristic for transplantation therapy, appears to be maintained when the stem cell characteristics of UCMSC are intact.

\section{UCMSC HOME TO TUMOR TISSUES}

MSC possess tropism to inflammatory tissues such as cancer tissues [28-31]. Both human and rat UCMSC also possess a strong tropism to various tumor tissues $[32,33]$ and other inflammatory lesions $[17,34,35]$. Both human and rat UCMSC were specifically localized in the periphery of the tumor tissues when they were transplanted (Fig. 1) $[32,33]$. The homing ability of stem cells seems to be mediated by the interaction of cytokines/growth factors and their receptors [36]. Large amounts of various cytokines and growth factors are secreted by tumor cells and their associated stroma $[37,38]$. Since UCMSC and other MSC express various cytokine and growth factor receptors on their membrane surfaces, they are likely to migrate towards cytokine/growth factor production sites by sensing these cytokine gradients $[14,31,39,40]$. For example, a subpopulation of BMMSC was shown to express CXCR4, the receptor for stromal cell-derived factor 1 (SDF-1), and CXCR3, the receptor for fractaline [41]. Tumors secrete SDF-1, PDGF, EGF, etc. that attract cells from surrounding tissues as well as from the bone marrow to provide support of tumor growth. Due to the over-expression of IL-8 receptor, CXCR1, and CXCR2, UCMSC have a greater capacity to migrate towards glioma than BMMSC [39]. In NPSC, which also exhibit tropism to gliomas, hypoxia is another key factor affecting tropism. SDF-1/CXCR4, uPA/uPAR, VEGF/VEGFR2, and hepatocyte growth factor/c-Met signaling pathways mediate increased NPSC-to-glioma tropism under hypoxic conditions [11]. However, the effect of hypoxia on UCMSC tumor tropism has not been reported. Hypoxic conditions may be one way in which tumor tissue provides a niche for stem cells. The tumor tropic properties of UCMSC and other MSC is apparently not disturbed by loading of various therapeutic genes [29, 42-45], drugs [46, 47], and nanoparticles into the cells. Thus, it is possible to utilize UCMSC as tumor or inflammatory tissue-targeted delivery vehicles for various therapeutics.

Table 1. Studies of Tumor Growth Attenuation by UCMSC and Other MSC. This Summary Table Lists Only Studies Describing Tumor Cell Growth Attenuation by UCMSC and Other MSC [15, 32,33,45,49,50,59-61]

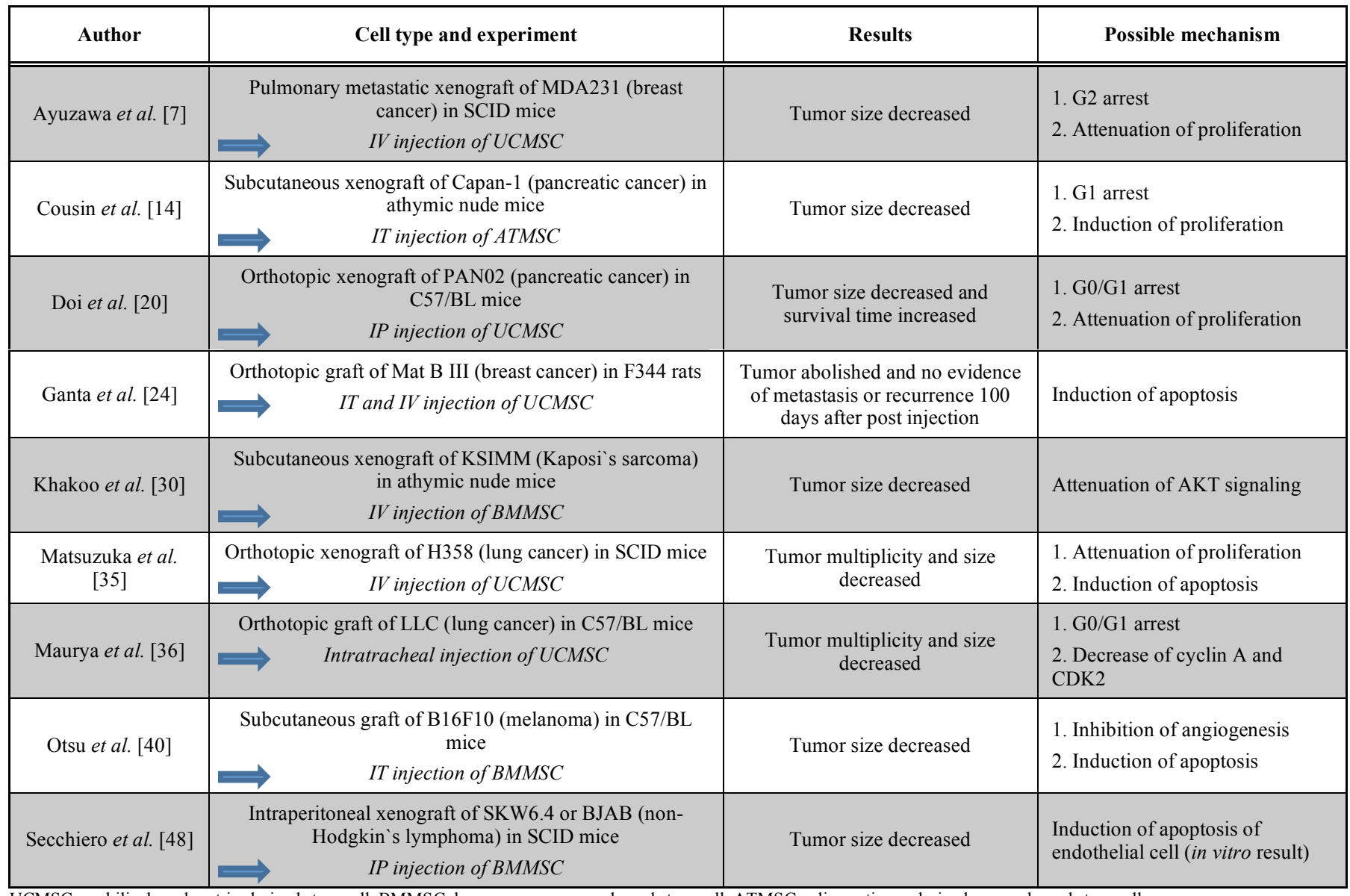

UCMSC: umbilical cord matrix-derived stem cell, BMMSC: bone marrow mesenchymal stem cell, ATMSC: adipose tissue-derived mesenchymal stem cell. 


\section{TUMORICIDAL ABILITY OF UCMSC}

Although many studies have demonstrated that MSC can have a stimulatory effect on tumor growth [48], naïve MSC, including UCMSC, BMMSC, and ATMSC, have been shown to be capable of attenuating the growth of tumor cells both in vitro and in vivo (Table 1). Both un-engineered human and rat UCMSC significantly attenuated growth of cancer cell lines in vitro and in vivo with human breast cancer cells [32], rat mammary tumor cells (Fig. 2) [49], human lung cancer cells (Fig. 4) [45], mouse Lewis lung carcinoma (LLC) cells [33], and mouse pancreatic carcinoma cells [33]. Detailed analysis with multiple assay methods including MTT assay, thymidine uptake assay, direct cell counting, and tumor colony assay clarified that this UCMSC-dependent growth attenuation is mediated by secretory proteins/peptides and is associated with decreased MAP kinase activity. The secretory proteins/peptides are produced by either the UCMSC or the tumor cells in response to the UCMSC secretory products. Analysis with flow cytometry further revealed that co-culture with human UCMSC caused G2 arrest of MDA 231 human breast cancer cells [32], whereas rat UCMSC caused G0/G1 arrest of LLC [50] and murine pancreatic carcinoma cells [33]. The G0/G1 arrest is evident in the decrease of CDK2 expression [50]. In vivo studies showed that both human and rat UCMSC treatment significantly decreased tumor weights $[32,33,49$, 50]. In particular, intratumoral or systemic administration of rat UCMSC caused complete regression of orthotopic Mat B III grafts in the female F344 rat mammary gland to undetectable levels by 34 to 38 days (Fig. 2). There was no evidence of metastasis or recurrence 100 days post-tumor cell inoculation (Fig. 2). Histological studies revealed that intratracheally, intraperitoneally, or systemically administered rat UCMSC homed to tumor areas and survived for at least 3 weeks without any evidence of differentiation or adverse effects $[33,50]$. Therefore, the findings described above suggest that UCMSC may represent a new therapeutic modality and will have important implications for the treatment of patients with breast cancer, lung cancer, pancreatic cancer, and other types of cancer.

\section{POSSIBLE MECHANISMS BY WHICH UCMSC ATTENUATE TUMOR GROWTH}

The mechanisms by which naïve UCMSC attenuate tumor growth have yet to be fully clarified, however, two potential mechanisms are suggested.

The first potential mechanism is that the UCMSC produce multiple secretory proteins that induce cell death of cancer cells and cell cycle arrest. This possibility is suggested by multiple reports that UCMSC stimulate caspase activities and arrest the cell cycle even in the absence of direct contact with cancer cells [7, 18, 22, 31]. In support of this mechanism, our recent study via microarray analysis of rat UCMSC discovered over-expression of multiple tumor suppressor genes when they were co-cultured with Mat B III rat mammary tumor cells in a Transwell culture system. In this culture system, the two cell types were divided by a porous membrane with $0.4 \mu \mathrm{m}$ pores and were devoid of direct contact. The comparison of gene expression profiles between untreated and co-cultured rat UCMSC identified five up-regulated genes (follistatin (FST), sulfatase1 (SULF1), glucose phosphate isomerase (GPI), HtrA serine peptidase (HTRA1), and adipocyte differentiation-related protein (ADRP)) and two down-regulated genes (transforming growth factor, beta-induced, $68 \mathrm{kDa}$ (TGFBI) and podoplanin (PDPN)) [51].

The second potential mechanism of tumor attenuation is the enhancement of an immune reaction to cancer cells by a mixture of UCMSC, malignant tumor cells, and dead tumor cells. Histopathology of the tumors of orthotopic Mat B III grafts in the female F344 rat mammary gland treated with rat UCMSC indicated that a large portion of tumor was replaced with extracellular matrix, and a large number of lymphocytes had infiltrated into tumor tissues (Fig. 3). Immunohistoche-
A

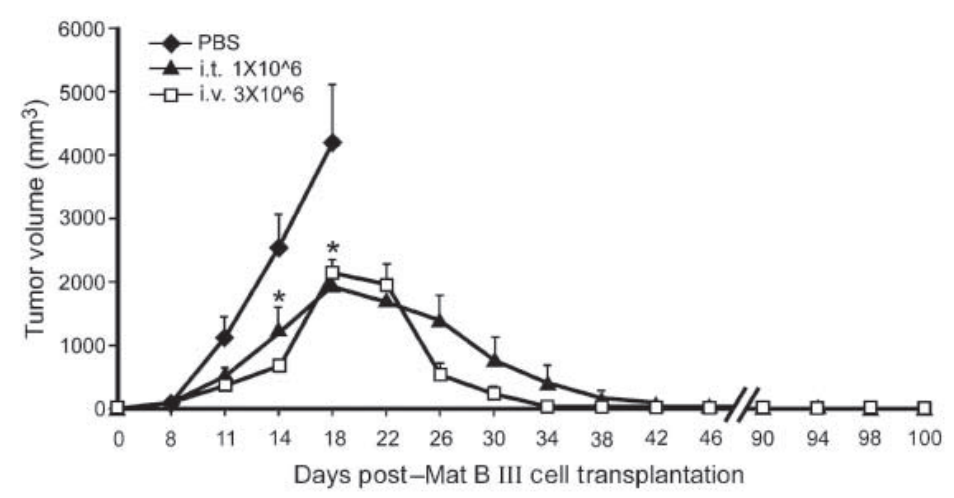

B

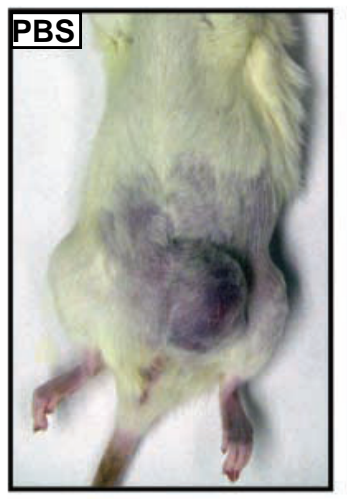

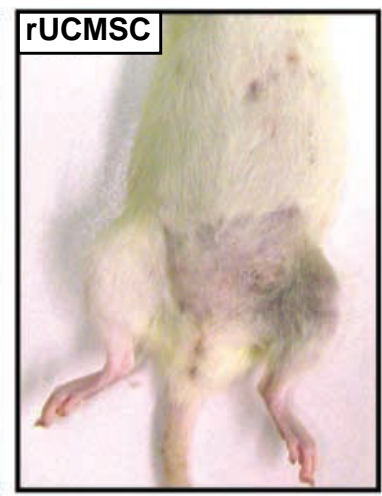

Fig. (2). Rat UCMSC completely eliminate mammary tumors without recurrence. Figure 2A shows Mat B III mammary tumor growth (volume) with time. Mat B III mammary tumor-bearing F344 rats were treated with rat UCMSC, either systemically via IV injection (1.5 x $10^{6}$ cells, at days 4 and 6 post tumor cell transplantation) or intratumorally (IT, $1 \times 10^{6}$ cells, bolus injection at day 4 post tumor cell transplantation). Rats bearing tumors reaching beyond $2 \pm 0.2 \mathrm{~cm}$ in diameter were sacrificed. *Significantly different from PBS treated rats. Figure 2B shows representative examples of rat mammary tumors after treatment with PBS or IV rat UCMSC. Figure is modified from the original figure, Ganta et al., Cancer Res, 69: 1815-1820, 2009 [49]. 
A
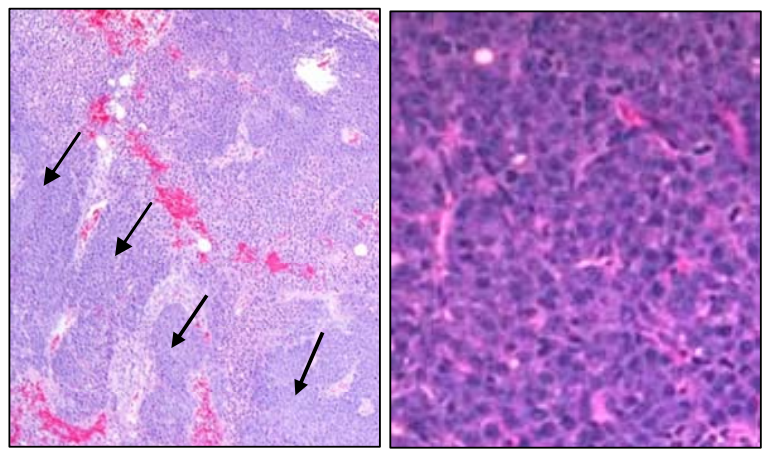

B

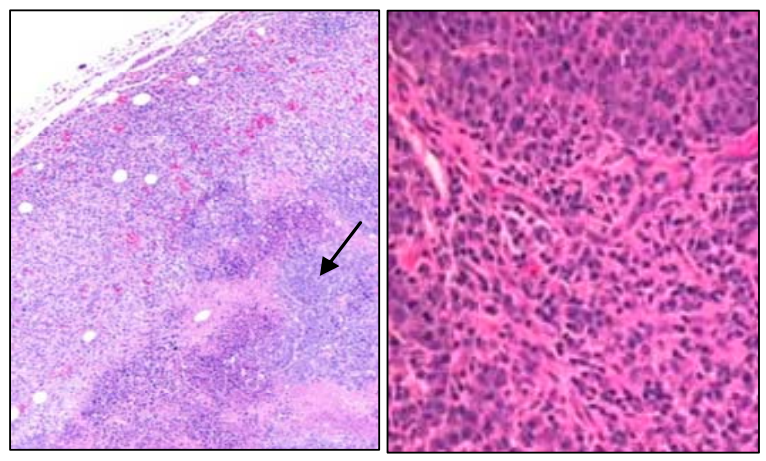

Fig. (3). Histopathologic analysis of orthotopic Mat B III mammary tumor grafts revealed that rat UCMSC treatment stimulates lymphocyte infiltration in the tumors. Control PBS treated mammary tumor grafts in female F344 rats contained mostly anaplastic tumor cells (arrows) (A), whereas rat UCMSC-treated rat tumors contained island-like clusters of tumor cells (arrow) surrounded by connective tissues with moderate to large numbers of lymphocytes (B). Figure is modified from the original figure, Ganta et al., Cancer Res, 69:1815-1820, 2009 [49].

mistry revealed that the majority of infiltrating lymphocytes in rat UCMSC-treated tumors were T cells. The treatment of rat UCMSC apparently increased CD $8+\mathrm{T}$ cell infiltration throughout the tumor tissue. CD68+ cells were occasionally observed in the tumors of the PBS control group but not observed in the rat UCMSC treated group. DNA microarray analysis suggested that monocyte chemotactic protein-1 (MCP-1 also known as CCL2), which is associated with lymphocyte infiltration, was the most likely candidate to induce migration of lymphocytes by rat UCMSC treatment. Although these results contradict results described above which show the low immunogenicity of human UCMSC, the immunogenicity of UCMSC in tumor-bearing animals may be dependent upon the microenvironment of UCMSC and tumor cells.

\section{UCMSC AS CELLULAR VEHICLES FOR TARGETED CANCER THERAPY}

Developing targeted cancer therapy requires cancer tissue-targeted delivery of therapeutics such as tumor suppressor genes, anticancer drugs, and/or therapeutic nanoparticles. UCMSC are very durable, have a large loading capacity, and have marked tumor tissue tropism. In addition, human UCMSC are useful human postnatal stem cells because a relatively large number can be harvested, propagated without any feeder cells, and stored after birth without any risks to the donor. As a result, they are ideal vehicles for targeted therapy. In fact, our previous reports have proven that human UCMSC are suited for tumortargeted delivery of various anticancer agents, such as the

A
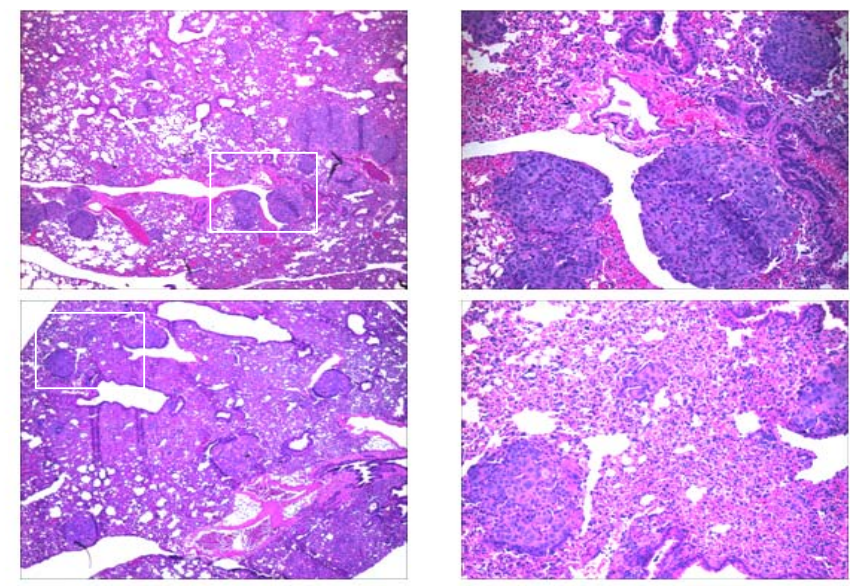

IFN $\beta$-hUCMSC

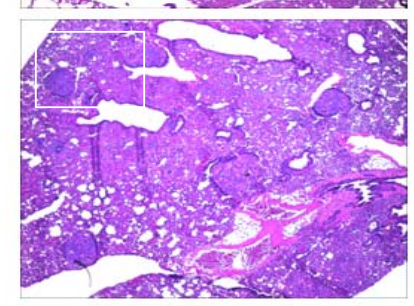

hUCMSC

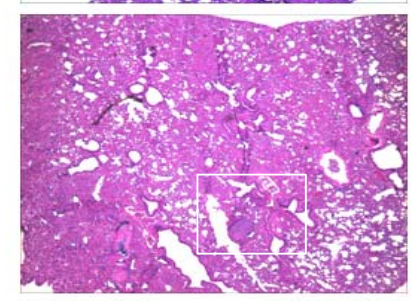

$50 x$

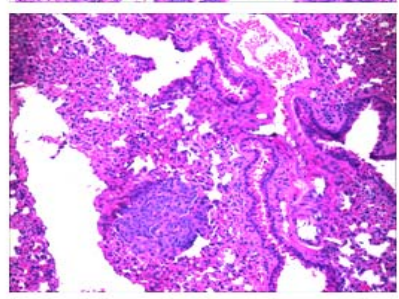

200x 
B

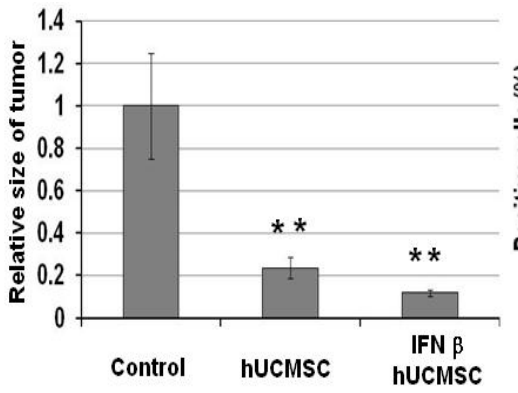

C

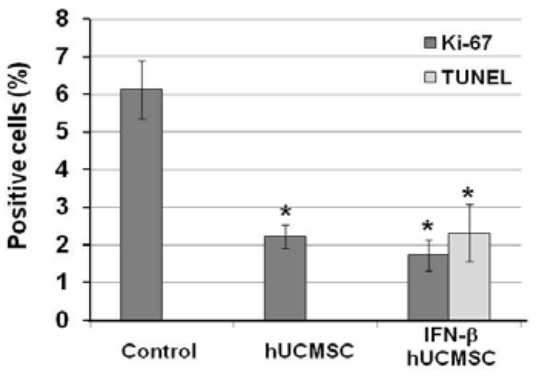

Fig. (4). IFN- $\beta$ expressing human UCMSC treatment significantly attenuates the growth of orthotopic lung adenocarcinoma xenografts. H358 orthotopic lung adenocarcinoma xenografts were treated systemically via IV injection with IFN- $\beta$-hUCMSC (4 x $10^{5}$ cells) four times with 5-day intervals. Two weeks after the last treatment, lungs were dissected and fixed in 10\% formalin. Paraffin embedded lung sections were stained by H\&E staining. Morphologies of lung tumors in three different treatments are presented in the upper panels (A). Multiple sizes of tumors, from as small as a cluster of several cancer cells to relatively large tumors, were counted under the microscope at 50x magnification. White squares in the 50x pictures indicate the area magnified in the 200x pictures. The relative tumor size (B), cell proliferation (Ki-67), and apoptosis (TUNEL) index (C) were presented in the bar graphs. ${ }^{*}, \mathrm{p}<0.05$ as compared to the level of PBS-treated control; **, ${ }^{*}<0.01$ as compared to the level of PBS-treated control. Figure is modified from the original figure, Matsuzuka et al., Lung Cancer, 70: 28-36, 2010.

cytotoxic cytokine interferon (IFN)- $\beta \quad[42,45]$ (Fig. 4) and the anticancer prodrug irinotecan (unpublished results). Our recent study has demonstrated that human UCMSC do not form any teratomas when injected into SCID mice [42]. Other types of MSC, such as BMMSC [29,30], adipose tissue-derived mesenchymal stem cells (ATMSC), and neuronal stem/progenitor cells (NSPC) are also shown to be usable for cancer-targeted delivery of various therapeutics $[52,53]$. Such applications cause significant attenuation of the growth of difficult tumors such as malignant glioma, metastatic melanoma, etc.

The primary benefits of live cell-based delivery of these therapeutics include a reduction of side effects and increased efficacy of each therapeutic agent. For example, the use of adenoviral vectors is an effective strategy as they allow strong expression of transgenes in a variety of tissues, including tumor tissue $[54,55]$. However, their clinical applications are limited by their efficacy in that viral vectors are rapidly cleared from circulation [56]. Viral vectors are also potentially pathogenic, as cases of viral infection have been reported [57]. Since naked plasmid DNA does not easily penetrate cellular membranes, non-viral gene delivery systems, such as nanoparticle encapsulation of therapeutic genes, may be an alternative procedure. However, since nanoparticles do not have tumor tropism, surface modifications that cause tumor-targeted accumulation are required. Loading nanoparticles encapsulating therapeutic genes or drugs into the stem cells and having these stem cells deliver the therapeutics to cancer tissues is a potentially viable alternative procedure.

IFN- $\beta$ is known to have a strong ability to inhibit tumor cell growth and induce apoptosis in vitro. However, IFN- $\beta$

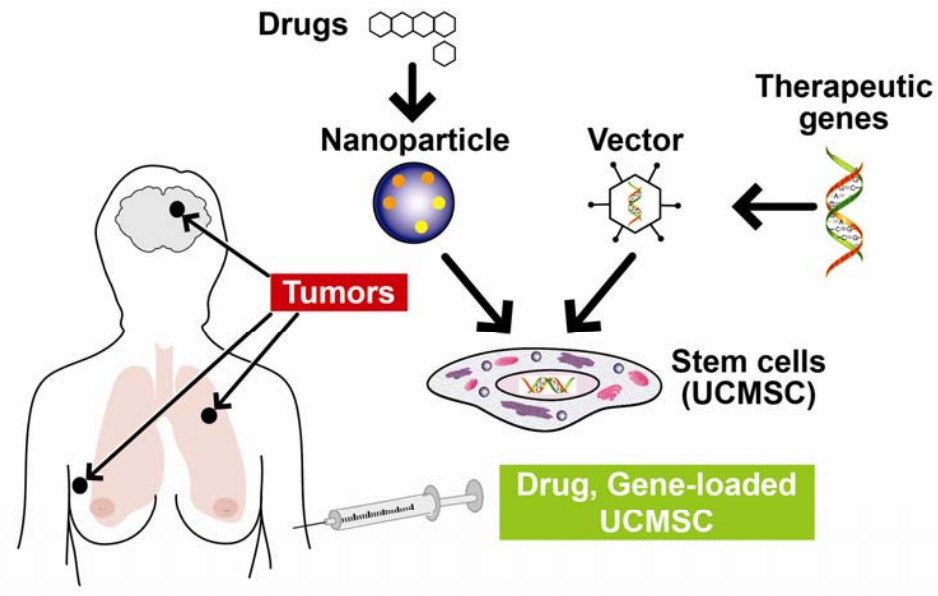

Fig. (5). Schematic illustration of stem cell-based cancer-targeted therapy. Systemically administered stem cells transfected with therapeutic genes or loaded with anticancer drugs/nanoparticles specifically migrate to the cancer site and produce cytotoxic cytokines or release active drugs/therapeutic nanoparticles effectively. This procedure overcomes short half-life of cytokine and side effects to normal tissues. 
has not been successfully used in in vivo studies because of its short half-life and because the maximally tolerated dose is lower than the effective dose. Recent studies by our colleagues, Rachakatla et al., and Matsuzuka et al., have shown successful expression of IFN- $\beta$ in human UCMSC (IFN- $\beta$-hUCMSC). They have demonstrated that systemically administered IFN- $\beta$-hUCMSC successfully homed to lung tumor sites and attenuated growth of lung-metastasized breast cancer [19] and human bronchioloalveolar carcinoma in mice (Fig. 4) [45]. A unique feature of these IFN- $\beta$ hUCMSC is that the efficacy of IFN- $\beta$-dependent tumor growth attenuation appears to be significantly enhanced by the intrinsic tumoricidal ability of the human UCMSC themselves [32]. Furthermore, poor immunogenicity [21, 58] and non-tumorigenicity [42] of human UCMSC make this cellular vehicle potentially applicable to human patients. This live cell-based cytokine gene therapy appears to be significantly safe and effective in controlling NSCLC, lung metastasis of other types of cancers, and various other types of primary cancers (Fig. 5). Cell-based cytokine gene and nanoparticle therapy should also be applicable to various inflammatory diseases.

\section{CONCLUSION}

Naïve UCMSC have many potential advantages for celldirected cancer therapy, since they have been shown to have the intrinsic ability to secrete factors that can result in cancer cell growth inhibition and/or apoptosis in vitro and in vivo. Among many tissue-originated multipotent stem cells, UCMSC are very usable due to their abundance, low immunogenicity, lack of CD34 and CD45 expression, and simplicity of the methods for harvest and in vitro expansion. These properties of UCMSC encourage their development as therapeutic tools or agents because they can potentially be used for allogeneic transplantation. In addition, human UCMSC engineered to express IFN- $\beta$ produced sufficient amounts of IFN- $\beta$ to induce death of human breast adenocarcinoma cells and bronchioloalveolar carcinoma cells in vitro and in vivo. Thus, the IFN- $\beta$-human UCMSC could also be a new therapeutic modality for the treatment of breast adenocarcinoma and bronchioloalveolar carcinoma and will have important implications for patients with breast cancer, lung cancer, or other types of cancers.

\section{ACKNOWLEDGEMENT}

The authors thank Ms. Marla Pyle and Ms. Jamie Ball (Department of Anatomy and Physiology, Kansas State University) for critical reading and constructive comments during the preparation of the manuscript. This work was supported in part by the Kansas State University (KSU) Terry C. Johnson Center for Basic Cancer Research, KSU College of Veterinary Medicine Dean's fund, KSU Targeted Excellence Research grant, Kansas State Legislative Appropriation, the Joan's Legacy Foundation Research Grant, NIH grants 1R21CA135599, P20 RR017686, P20 RR016475, P20RR015563, Kansas Bioscience Authority Collaborative Cancer Research Grant and the Intramural Research Program of the NIH, National Institute on Aging.

\section{CONFLICT OF INTEREST}

None declared.

\section{ABBREVIATIONS}

\begin{tabular}{|c|c|c|}
\hline UCMSC & $=$ & Umbilical cord matrix stem cells \\
\hline $\mathrm{MSC}$ & $=$ & Mesenchymal stem cells \\
\hline BMMSC & $=$ & Bone marrow mesenchymal stem cells \\
\hline ATMSC & $=$ & $\begin{array}{l}\text { Adipose tissue-derived mesenchymal } \\
\text { stem cells }\end{array}$ \\
\hline NSPC & $=$ & Neuronal stem/progenitor cells \\
\hline PBMC & $=$ & Peripheral blood mononuclear cells \\
\hline IL-6 & $=$ & Interleukin-6 \\
\hline IL-10 & $=$ & Interleukin-10 \\
\hline TGF- $\beta$ & $=$ & Transforming growth factor-beta \\
\hline HLA-ABC & $=$ & Human leukocyte antigen-ABC \\
\hline HLA-DR & $=$ & Human leukocyte antigen-DR \\
\hline HLA-G & $=$ & Human leukocyte antigen-G \\
\hline Treg & $=$ & Regulatory T cells \\
\hline ICAM-1 & $=$ & $\begin{array}{l}\text { Inflammatory cytokine-induced intercellular } \\
\text { adhesion molecule-1 }\end{array}$ \\
\hline VCAM-1 & $=$ & Vascular cell adhesion molecule-1 \\
\hline SDF-1 & $=$ & Stromal cell-derived factor 1 \\
\hline MTT & $=$ & $\begin{array}{l}\text { 3-(4,5-Dimethylthiazol-2-yl)-2,5- } \\
\text { diphenyltetrazolium }\end{array}$ \\
\hline IFN- $\beta$ & $=$ & Interferon- $\beta$ \\
\hline IFN- $\gamma$ & $=$ & Interferon- $\gamma$ \\
\hline EGF & $=$ & Epidermal growth factor \\
\hline PDGF & $=$ & Platelet-derived growth factor \\
\hline VEGF & $=$ & Vascular endothelial cell growth factor \\
\hline CXCR3 & $=$ & $\begin{array}{l}\text { C-X-C chemokine receptor type } 3 \text { for } \\
\text { CXCL9, CXCL10 and CXCL11 }\end{array}$ \\
\hline CXCR4 & $=$ & $\begin{array}{l}\text { C-X-C chemokine receptor type } 4 \text { for } \\
\text { stromal cell-derived factor } 1 \text { (SDF-1)IV, } \\
\text { intravenous }\end{array}$ \\
\hline IT & $=$ & Intratumoral \\
\hline IP & $=$ & Intraperitoneal \\
\hline
\end{tabular}

\section{REFERENCES}

[1] Kobayashi K, Kubota T, Aso T. Study on myofibroblast differentiation in the stromal cells of Wharton's jelly: expression and localization of alpha-smooth muscle actin. Early Hum Dev 1998; 51: 223-33.

[2] De Miguel MP, Arnalich Montiel F, Lopez Iglesias P, Blazquez Martinez A, Nistal M. Epiblast-derived stem cells in embryonic and adult tissues. Int J Dev Biol 2009; 53: 1529-40.

[3] Pappa KI, Anagnou NP. Novel sources of fetal stem cells: where do they fit on the developmental continuum? Regen Med 2009; 4: 423-33.

[4] Carlin R, Davis D, Weiss M, Schultz B, Troyer D. Expression of early transcription factors Oct4, Sox 2 and Nanog by porcine 
umbilical cord (PUC) matrix cells. Reprod Biol Endocrinol 2006; 4: 8 .

[5] La Rocca G, Anzalone R, Corrao S, et al. Isolation and characterization of Oct-4+/HLA-G+ mesenchymal stem cells from human umbilical cord matrix: differentiation potential and detection of new markers. Histochem Cell Biol 2009; 131: 267-82.

[6] Mitchell KE, Weiss ML, Mitchell BM, et al. Matrix cells from Wharton's jelly form neurons and glia. Stem Cells 2003; 21: 50-60.

[7] Wang HS, Hung SC, Peng ST, et al. Mesenchymal stem cells in the Wharton's jelly of the human umbilical cord. Stem Cells 2004; 22: 1330-7.

[8] Karnoub AE, Dash AB, Vo AP, et al. Mesenchymal stem cells within tumour stroma promote breast cancer metastasis. Nature 2007; 449: 557-63.

[9] Yu JM, Jun ES, Bae YC, Jung JS. Mesenchymal stem cells derived from human adipose tissues favor tumor cell growth in vivo. Stem Cells Dev 2008; 17: 463-73.

[10] Galiè M, Konstantinidou G, Peroni D, et al. Mesenchymal stem cells share molecular signature with mesenchymal tumor cells and favor early tumor growth in syngeneic mice. Oncogene 2008; 27 : 2542-51.

[11] Ren G, Zhang L, Zhao X, et al. Mesenchymal stem cell-mediated immunosuppression occurs via concerted action of chemokines and nitric oxide. Cell Stem Cell 2008; 2: 141-50.

[12] Kinnaird T, Stabile E, Burnett MS, et al. Marrow-derived stromal cells express genes encoding a broad spectrum of arteriogenic cytokines and promote in vitro and in vivo arteriogenesis through paracrine mechanisms. Circ Res 2004; 94: 678-85.

[13] Martin FT, Dwyer RM, Kelly J, et al. Potential role of mesenchymal stem cells (MSCs) in the breast tumour microenvironment: stimulation of epithelial to mesenchymal transition (EMT). Breast Cancer Res Treat 2010; 124: 317-26.

[14] Djouad F, Plence P, Bony C, et al. Immunosuppressive effect of mesenchymal stem cells favors tumor growth in allogeneic animals. Blood 2003; 102: 3837-44.

[15] Khakoo AY, Pati S, Anderson SA, et al. Human mesenchymal stem cells exert potent antitumorigenic effects in a model of Kaposi's sarcoma. J Exp Med 2006; 203: 1235-47.

[16] Yang CC, Shih YH, Ko MH, et al. Transplantation of human umbilical mesenchymal stem cells from Wharton's jelly after complete transection of the rat spinal cord. PLoS One 2008; 3: e3336.

[17] Xiong N, Zhang Z, Huang J, et al. VEGF-expressing human umbilical cord mesenchymal stem cells, an improved therapy strategy for Parkinson's disease. Gene Ther 2010; 18: 394-402.

[18] Chao KC, Chao KF, Fu YS, Liu SH. Islet-like clusters derived from mesenchymal stem cells in Wharton's Jelly of the human umbilical cord for transplantation to control type 1 diabetes. PLoS ONE 2008; 3: e1451.

[19] Rachakatla RS, Pyle MM, Ayuzawa R, et al. Combination treatment of human umbilical cord matrix stem cell-based interferon-beta gene therapy and 5-fluorouracil significantly reduces growth of metastatic human breast cancer in SCID mouse lungs. Cancer Invest 2008; 26: 662-70.

[20] Prasanna SJ, Gopalakrishnan D, Shankar SR, Vasandan AB. Proinflammatory cytokines, IFNgamma and TNFalpha, influence immune properties of human bone marrow and Wharton jelly mesenchymal stem cells differentially. PLoS One 2010; 5: e9016.

[21] Weiss ML, Anderson C, Medicetty S, et al. Immune properties of human umbilical cord Wharton's jelly-derived cells. Stem Cells 2008; 26: 2865-74.

[22] Deuse T, Stubbendorff M, Tang-Quan K, et al. Immunogenicity and immunomodulatory properties of umbilical cord lining mesenchymal stem cells. Cell Transplant 2010.

[23] Selmani Z, Naji A, Zidi I, et al. Human leukocyte antigen-G5 secretion by human mesenchymal stem cells is required to suppress $\mathrm{T}$ lymphocyte and natural killer function and to induce CD4+CD25highFOXP3+ regulatory T cells. Stem Cells 2008; 26: 212-22.

[24] Griffin MD, Ritter T, Mahon BP. Immunological aspects of allogeneic mesenchymal stem cell therapies. Hum Gene Ther 2010; 21: $1641-55$.

[25] Cutler AJ, Limbani V, Girdlestone J, Navarrete CV. Umbilical cord-derived mesenchymal stromal cells modulate monocyte function to suppress T cell proliferation. J Immunol 2010; 185: 6617-23.
[26] Wang D, Chen K, Du WT, et al. CD14+ monocytes promote the immunosuppressive effect of human umbilical cord matrix stem cells. Exp Cell Res 2010; 316: 2414-23.

[27] Chen X, McClurg A, Zhou GQ, et al. Chondrogenic differentiation alters the immunosuppressive property of bone marrow-derived mesenchymal stem cells, and the effect is partially due to the upregulated expression of B7 molecules. Stem Cells 2007; 25: 364 70.

[28] Aboody KS, Brown A, Rainov NG, et al. Neural stem cells display extensive tropism for pathology in adult brain: evidence from intracranial gliomas. Proc Natl Acad Sci U S A 2000; 97: 1284651.

[29] Studeny M, Marini FC, Champlin RE, et al. Bone marrow-derived mesenchymal stem cells as vehicles for interferon-beta delivery into tumors. Cancer Res 2002; 62: 3603-8.

[30] Studeny M, Marini FC, Dembinski JL, et al. Mesenchymal stem cells: potential precursors for tumor stroma and targeted-delivery vehicles for anticancer agents. J Natl Cancer Inst 2004; 96: 1593603.

[31] Nakamizo A, Marini F, Amano T, et al. Human bone marrowderived mesenchymal stem cells in the treatment of gliomas. Cancer Res 2005; 65: 3307-18.

[32] Ayuzawa R, Doi C, Rachakatla RS, et al. Naive human umbilical cord matrix derived stem cells significantly attenuate growth of human breast cancer cells in vitro and in vivo. Cancer Lett 2009; 280: 31-7.

[33] Doi C, Maurya DK, Pyle MM, Troyer D, Tamura M. Cytotherapy with naive rat umbilical cord matrix stem cells significantly attenuates growth of murine pancreatic cancer cells and increases survival in syngeneic mice. Cytotherapy 2010; 12: 408-17.

[34] Dvorak HF. Tumors: wounds that do not heal. Similarities between tumor stroma generation and wound healing. . N Engl J Med 1986; 315: $1650-59$.

[35] Zhu Y, Sun Z, Han Q, et al. Human mesenchymal stem cells inhibit cancer cell proliferation by secreting DKK-1. Leukemia 2009; 23: 925-33.

[36] Muller A, Homey B, Soto H, et al. Involvement of chemokine receptors in breast cancer metastasis. Nature 2001; 410: 50-6.

[37] Hall B, Andreeff M, Marini F. The participation of mesenchymal stem cells in tumor stroma formation and their application as targeted-gene delivery vehicles. Handb Exp Pharmacol 2007; 26383 .

[38] Arbab AS, Janic B, Knight RA, et al. Detection of migration of locally implanted AC133+ stem cells by cellular magnetic resonance imaging with histological findings. Faseb J 2008; 22: 3234-46.

[39] Kim DS, Kim JH, Lee JK, et al. Overexpression of CXC chemokine receptors is required for the superior glioma-tracking property of umbilical cord blood-derived mesenchymal stem cells. Stem Cells Dev 2009; 18: 511-9.

[40] Park SA, Ryu CH, Kim SM, et al. CXCR4-transfected human umbilical cord blood-derived mesenchymal stem cells exhibit enhanced migratory capacity toward gliomas. Int J Oncol 2011; 38: 97-103.

[41] Yu J, Li M, Qu Z, et al. SDF-1/CXCR4-mediated migration of transplanted bone marrow stromal cells toward areas of heart myocardial infarction through activation of PI3K/Akt. J Cardiovasc Pharmacol 2010; 55: 496-505.

[42] Rachakatla RS, Marini F, Weiss ML, Tamura M, Troyer D. Development of human umbilical cord matrix stem cell-based gene therapy for experimental lung tumors. Cancer Gene Ther 2007; 14: 828-35.

[43] Reddig PJ, Dreckschmidt N, et al. Transgenic mice overexpressing protein kinase Cdelta in the epidermis are resistant to skin tumor promotion by 12-O-tetradecanoylphorbol-13-acetate. Cancer Res 1999; 59: 5710-18.

[44] Dembinski JL, Spaeth EL, Fueyo J, et al. Reduction of nontarget infection and systemic toxicity by targeted delivery of conditionally replicating viruses transported in mesenchymal stem cells. Cancer Gene Ther 2009; 17: 289-97.

[45] Matsuzuka T, Rachakatla RS, Doi C, et al. Human umbilical cord matrix-derived stem cells expressing interferon-beta gene significantly attenuate bronchioloalveolar carcinoma xenografts in SCID mice. Lung Cancer 2010; 70: 28-36. 
[46] Frank RT, Edmiston M, Kendall SE, et al. Neural stem cells as a novel platform for tumor-specific delivery of therapeutic antibodies. PLoS One 2009; 4: e8314.

[47] Gutova M, Najbauer J, Chen MY, et al. Therapeutic targeting of melanoma cells using neural stem cells expressing carboxylesterase, a CPT-11 activating enzyme. Curr Stem Cell Res Ther 2010; 5: 273-6.

[48] Klopp AH, Gupta A, Spaeth E, et al. Concise review: Dissecting a discrepancy in the literature: do mesenchymal stem cells support or suppress tumor growth? Stem Cells 2011; 29: 11-9.

[49] Ganta C, Chiyo D, Ayuzawa R, et al. Rat umbilical cord stem cells completely abolish rat mammary carcinomas with no evidence of metastasis or recurrence 100 days post-tumor cell inoculation. Cancer Res 2009; 69: 1815-20.

[50] Maurya DK, Doi C, Kawabata A, et al. Therapy with unengineered naive rat umbilical cord matrix stem cells markedly inhibits growth of murine lung adenocarcinoma. BMC Cancer 2010; 10: 590

[51] Doi C, Egashira N, Kawabata A, et al. Angiotensin II type 2 receptor signaling significantly attenuates growth of murine pancreatic carcinoma grafts in syngeneic mice. BMC Cancer 2010; 67.

[52] Aboody KS, Najbauer J, Schmidt NO, et al. Targeting of melanoma brain metastases using engineered neural stem/progenitor cells. Neuro Oncol 2006; 8: 119-26.
[53] Aboody KS, Najbauer J. Danks MK. Stem and progenitor cellmediated tumor selective gene therapy. Gene Ther 2008; 15: 73952.

[54] Simons M, Beinroth S, Gleichmann M, et al. Adenovirus-mediated gene transfer of inhibitors of apoptosis protein delays apoptosis in cerebellar granule neurons. J Neurochem 1999; 72: 292-301.

[55] Benihoud K, Yeh P, Perricaudet M. Adenovirus vectors for gene delivery. Curr Opin Biotechnol 1999; 10: 440-7.

[56] Csikos T, Gallinat SUnger T. Extrarenal aspects of angiotensin II function. Eur J Endocrinol 1997; 136: 349-58.

[57] Ragusa A, Garcia I, Penades S. Nanoparticles as nonviral gene delivery vectors. IEEE Trans Nanobioscience 2007; 319-30.

[58] Cho PS, Messina DJ, Hirsh EL, et al. Immunogenicity of umbilical cord tissue derived cells. Blood 2008; 111: 430-8.

[59] Cousin B, Ravet E, Poglio S, et al. Adult stromal cells derived from human adipose tissue provoke pancreatic cancer cell death both in vitro and in vivo. PLoS One 2009; 4: e6278.

[60] Otsu K, Das S, Houser SD, Quadri SK, Bhattacharya S, Bhattacharya J. Concentration-dependent inhibition of angiogenesis by mesenchymal stem cells. Blood 2009; 113: 4197-205.

[61] Secchiero P, Zorzet S, Tripodo C, et al. Human bone marrow mesenchymal stem cells display anti-cancer activity in SCID mice bearing disseminated non-Hodgkin's lymphoma xenografts. PLoS One 2010; 5: e11140.

Received: March 09, 2011

Revised: May 16, 2011

Accepted: June 12, 2011

(C) Tamura et al.; Licensee Bentham Open.

This is an open access article licensed under the terms of the Creative Commons Attribution Non-Commercial License (http://creativecommons.org/ licenses/by-nc/3.0/), which permits unrestricted, non-commercial use, distribution and reproduction in any medium, provided the work is properly cited. 